\title{
Derechos Humanos: trabajo y seguridad social frente a las nuevas tecnologías de la cuarta revolución industrial en México*
}

\section{Emmanuel López Pérez**}

\section{RESUMEN}

El presente artículo tiene como objetivo el análisis del impacto de las nuevas tecnologías de la cuarta revolución industrial en dos grandes derechos humanos: trabajo y seguridad social.

Conscientes de que durante la evolución de las sociedades los avances tecnológicos han sido una constante en la vida cotidiana de las personas sin embargo, hoy en día esta $4 R \Gamma^{\prime \prime}$ generará cambios radicales por lo que estas situaciones de hecho deberán ser reguladas por un nuevo derecho.

En ese contexto este análisis se desarrollará mediante el uso de los métodos deductivo-analítico; primeramente se analizan las principales acepciones del tema, los efectos de las nuevas TICs en el trabajo y la seguridad social, el nuevo mercado laboral asi como los posibles esquemas de aseguramiento social del futuro. Por último, se presentan a manera de conclusiones retos que deberán afrontar las personas-Estados para la protección de estos derechos humanos en una era digital, finalizando con las fuentes de investigación.

\section{PALABRAS CLAVE}

Trabajo, seguridad social, cuarta revolución industrial, trabajador 4.0

\begin{abstract}
The main objective of this article is to analyze the impact of the new technologies of the fourth industrial revolution on two human rights: labor and social security.

Aware that during the evolution of societies technological advances have been a constant in people's daily lives, however, today this $4 R I$, will generate radical changes, so these situations must in fact be regulated by a new law.

In that context this analysis will be developed through the use of deductive-analytical methods; First, the main meanings of the topic, the effects of new TICS on labor and social security, the new labor market as well as the possible social insurance schemes of the future.

Finally, the main challenges in the matter are presented -in conclusion form-. and the sources of research
\end{abstract}

\section{KEYWORDS}

Labor, social security, fourth industrial revolution, worker 4.0

*Artículo de Investigación postulado el 10 de febrero de 2020 y aceptado el 4 de noviembre de 2020

**Doctorando en la Facultad de Derecho y Ciencias Sociales de la Universidad Autónoma del Estado de Morelos, México. (emmanuel.lopezper@uaem.edu.mx) orcid.org/0000-0003-2416-9292

***A partir de este momento se usará este Acrónimo para referirnos a la cuarta revolución industrial 


\section{SUMARIO}

1. Introducción

2. Precisiones terminológicas

3 La nueva revolución industrial

4 La industria 4.0 y el trabajo

5. El derecho a la seguridad social frente a la industria 4.0

6. Hacia una seguridad social 4.0

7. Reflexiones finales

\section{Introducción}

La evolución de las sociedades ha sido marcada por grandes avances tecnológicos que han roto paradigmas en diversos aspectos de las personas. Estos avances de las tecnologías se vieron representadas en tres grandes revoluciones industriales en donde cada una se caracterizó por diferentes invenciones.

En la actualidad nos enfrentamos a una cuarta revolución industrial o también llamada industria 4.0 la cual, se encuentra caracterizada por una completa automatización en los procesos de producción gracias a las inteligencias artificiales, las tecnologías digitales y el internet de las cosas.

Estas nuevas tecnologías que conlleva la industria 4.0 traen ciertas ventajas y desventajas en todos los aspectos de la vida cotidiana de las personas sin embargo, por ser interés principal del presente artículo se resalta aquellos que influyen directamente en dos derechos humanos importantes en México; el trabajo y la seguridad social.

En ese contexto el presente trabajo tiene como objetivo establecer cuál es el impacto de las nuevas tecnologías de la industria 4.0 en materia del trabajo y seguridad social, así como cuestionar los requerimientos o propuestas necesarias para hacer frente a estos nuevos hechos que deben ser regulados por un nuevo derecho.

Para ello, el presente artículo se desarrolla bajo el uso de los métodos deductivo-analítico; en un primer momento se establecen las precisiones terminológicas que envuelven al tema; a la postre, se analiza la nueva revolución industrial y sus antecedentes así como el impacto de esta $4 R I$ en el trabajo y la seguridad social, proponiendo el nacimiento de una seguridad social 4.0.

Por último, se desarrollan las reflexiones finales que se llegaron en la elaboración del presente trabajo. 


\section{Precisiones terminológicas}

\section{a. Trabajo}

El trabajo ha estado presente desde la existencia del hombre y la mujer sin embargo, la concepción del mismo ha evolucionado con base a los propios contextos y necesidades sociales que se desarrollan en determinada época.

De tal suerte que hablar de trabajo en la era primitiva no resulta igual a lo que fue en el siglo XVIII con la primera revolución industrial, pues basta señalar que nuestros ancestros desempeñaban trabajos mediante la división del mismo ${ }^{1}$ con la finalidad de sobrevivir a las propias inclemencias de la época sin tener como objetivo una retribución de naturaleza económica sino de primera necesidad. Por el contrario, hoy en día hablar de trabajo implica obligatoriamente tener como eje primordial una retribución reflejada tanto en dinero como en especie.

A pesar de ello, se debe resaltar que el trabajo sea la época en que se desarrolle tiene un elemento en común, su finalidad, la cual se encuentra direccionada en la satisfacción de las necesidades básicas de la persona y su entorno.

La existencia de múltiples acepciones de este concepto obliga a retomar aquellos que por su naturaleza fungen como un pilar para determinar lo que es el trabajo.

$\mathrm{Al}$ respecto, la Organización Internacional del Trabajo (OIT) establece que el trabajo es el conjunto de actividades humanas, remuneradas o no, que producen bienes o servicios en una economia, o que satisfacen las necesidades de una comunidad o proveen los medios de sustento necesarios para los individuos. ${ }^{2}$ Dentro de esta definición se encuentran inmersos elementos importantes tales como la actividad humana que puede ser remunerada o no, así como la producción de bienes y servicios que satisfagan las necesidades de los individuos dentro de su comunidad.

Por su parte, el Doctor Néstor de Buen en su libro "Derecho del trabajo" considera al trabajo como un sinónimo de actividad provechosa, de esfuerzo dirigido a la consecución de un fin valioso, ${ }^{3}$ desprendiendo que todo trabajo implica un esfuerzo físico o mental el cual, tiene como objetivo la obtención de una contraprestación de naturaleza económica o en especie.

\footnotetext{
1 División del trabajo como la recolección de frutos, la caza de animales, el cuidado de los hijos, entre otros. ${ }^{2}$ Organización Internacional del Trabajo, ¿Qué es el trabajo decente?, OIT, Suiza, 2004, [Consulta: 4 de enero, 2020]. Disponible en: https://www.ilo.org/americas/sala-de-prensa/WCMS_LIM_653_SP/lang--es/index.htm ${ }^{3}$ De Buen, Néstor, Derecho del Trabajo, Editorial Porrúa, México, 2015, p. 19.
} 
En ese contexto, la propia Ley Federal del Trabajo en su artículo octavo define como actividad humana, intelectual o material, independientemente del grado de preparación técnica requerido por cada profesión u oficio. ${ }^{4}$ Ciertamente la existencia de diversas acepciones respecto de un concepto tan controvertido como el trabajo, implica el estudio y contexto donde se encuentra implementado (resaltando por supuesto la época en que se vive).

De lo anterior surge el cuestionamiento respecto de la evolución del trabajo a causa de las nuevas tecnologías digitales de la $4 R I$.

El tiempo ha confirmado que la concepción del trabajo ha cambiado por lo tanto, resulta coherente establecer que el trabajo que hoy conocemos en diez o veinte años no será el mismo por lo que la definición que contempla la legislación laboral en el país tendrá que transformarse para encuadrar a la nueva realidad en la materia a causa de las tecnologías tanto en México como en el resto del mundo.

\section{b. Seguridad social}

El concepto seguridad social ha sufrido varias mutaciones a lo largo de la historia, en gran parte por los cambios socioculturales y económicos que sufren las sociedades pese a ello, su finalidad no ha logrado cambiar enfocándose a brindar una protección social a las personas con la intención de elevar su calidad de vida a través de sus principales herramientas: la beneficencia social, la asistencia social, el seguro social y los seguros privados.

La seguridad social es tan antigua como la humanidad, ${ }^{5}$ ya que los seres humanos por naturaleza buscamos tener una vida plena y lo más satisfactoria posible con los elementos presentes en nuestro entorno donde la seguridad social ha fungido como un medio para garantizar dicha plenitud.

$\mathrm{Al}$ respecto, la OIT refiere que la seguridad social:

Es la protección que la sociedad proporciona a sus miembros, bajo un enfoque integral de bienestar del trabajador y su familia, que consiste en proporcionar atención a las contingencias en materia de salud por enfermedad, maternidad y protección del trabajador en casos de accidente, desempleo, invalidez, vejez o muerte y el otorgamiento de prestaciones económicas y subsidios a la población con menores ingresos. ${ }^{6}$

\footnotetext{
${ }^{4}$ Ley Federal del Trabajo, Diario Oficial de la Federación, 1 de abril de 1970.

${ }^{5}$ Briseño Ruiz, Alberto, Derecho Mexicano de los Seguros Sociales, Ed. Harla, México, 2017, p.13.

${ }^{6}$ Organización Internacional del Trabajo, Indicadores del sistema seguridad social glosario de seguridad social y
} 
Como se desprende del concepto establecido por la OIT de una manera general, la seguridad social comprende diversas ramas de protección social enfocadas al trabajador y sus beneficiarios. Para ello se debe observar desde dos puntos de vista; el primero, garantizándolo a través de prestaciones de naturaleza económicas y; el segundo, mediante las prestaciones en especie que comúnmente derivan de la atención médica, hospitalaria, farmacéutica, sociales, entre otros.

En México la legislación no contempla un concepto referente a la seguridad social sin embargo, no resulta ser omisa del todo puesto que se establece su finalidad, al mencionar que:

La seguridad social tiene por finalidad garantizar el derecho a la salud, la asistencia médica, la protección de los medios de subsistencia y los servicios sociales necesarios para el bienestar individual y colectivo, así como el otorgamiento de una pensión que, en su caso y previo cumplimiento de los requisitos legales, será⿴ garantizada por el Estado. ${ }^{7}$

De tal suerte que la seguridad social garantiza los elementos sociales necesarios así como los económicos para que las personas puedan gozar de las prestaciones que ésta ofrece, aclarando que para ser sujeto de dichas prestaciones que la ley otorga se deberá cumplir con los requisitos que la misma exige.

Por consiguiente, se debe establecer que la seguridad social es:

El conjunto de instituciones, principios, normas y disposiciones que protegen a todas las personas contra cualquier contingencia que pudieran sufrir y las previene, a fin de permitir su bienestar mediante la superación de aspectos psicofísico, moral, económico, social y cultural. ${ }^{8}$

Ahora bien, se debe resaltar que la principal problemática de la seguridad social en México surge a causa del tratamiento dado por la legislación nacional ya que no es contemplada como un derecho humano sino correspondiente de otro derecho humano, el trabajo. Es decir la seguridad social en México se encuentra laboralizada, ya que sin la existencia de un trabajo formal el acceso a ella resulta compleja, ciertamente existe el aseguramiento voluntario a través del 
seguro de familia aunque, el acceso a las prestaciones solo se limita aquellas contempladas en la rama de enfermedad y maternidad.

Esta problemática se agudiza al empalmar las nuevas modalidades de trabajar a raíz de las tecnologías digitales de la $4 R I$ pues se espera que la implementación de inteligencias artificiales remplacen la mano de obra humana en los centros de trabajo ${ }^{9}$ originando dos hechos: la perdida de trabajos y por consiguiente el aseguramiento social.

De lo anterior la seguridad social no solo se limita a garantizar determinadas prestaciones pecuniarias y en especie a las personas, sino a la par funge como un estabilizador socioeconómico de un país. Sobra mencionar la gran importancia de este derecho humano en la vida de las personas contemplado en la Declaración Universal de los Derechos Humanos de la ONU en su artículo $22,{ }^{10}$ un derecho humano que hasta hoy su materialización ha sido un gran reto para la mayoría de los países de América Latina y México poniendo una carga más a los avances tecnológicos de la $4 R I$.

\section{c. Industria 4.0}

La concepción de la industria 4.0, también conocida como cuarta revolución industrial conlleva de manera implícita la aplicación y uso de nuevas tecnologías digitales en diversos aspectos de la vida de las personas (principalmente en aspectos que involucren el trabajo); cabe mencionar que contar con una definición precisa del significado de industria 4.0 resulta compleja, ya que hasta 2015 Carolina Castresna señaló que había más de 134 definiciones para poder explicar qué es este fenómeno global. ${ }^{11}$

No obstante, cada una de estas definiciones comparten algunas similitudes que permiten abrir un panorama respecto al significado de lo que es este fenómeno.

La industria 4.0 es:

Un nuevo modelo de organización y de control de la cadena de valor a través del ciclo de vida del producto y a lo largo de los sistemas de fabricación apoyado y hecho posible por las tecnologías de la información. ${ }^{12}$

\footnotetext{
${ }^{9}$ Se verá con mayor profundidad adelante.

${ }^{10}$ Naciones Unidas, Declaración Universal de los Derechos Humanos, 1948, Disponible en: https://www.un.org/es/ universal-declaration-human-rights/

${ }^{11}$ Cfr. Castresna Sáenz, Carolina, et al., Industria 4.0, España, Universidad de la Rioja, 2016, p. 12, [Consulta: 22 de diciembre, 2019]. Disponible en: https://biblioteca.unirioja.es/tfe_e/TFE002004.pdf

${ }^{12}$ Conferencia de Directores y Decanos de Ingeniería Informática, Industria 4.0: la transformación digital de la indus-
} 
Donde la interacción del hombre en los sistemas de producción se verá disminuido en razón a la automatización de los procesos originado por la implementación de inteligencias artificiales.

De tal suerte que esta industria 4.0 se encuentra basada en sistemas de producción ciberfísicos. En estos, los procesos de producción sean físicos o biológicos, son controlados o monitoreados por algoritmos estrechamente integrados con Internet. ${ }^{13}$

De una manera más digerible, se traduce que las tecnologías permitirán una interacción totalmente autónoma entre máquinas donde la mano del hombre se reducirá a lo mínimo, permitiendo una producción de los bienes y servicios con mayor eficacia y eficiencia gracias a los procesos digitales hechos posibles por el internet de las cosas. ${ }^{14}$

Sin duda, la industria 4.0 se:

Asocia con la informatización y digitalización de la producción y con la generación, integración y análisis de una gran cantidad de datos a lo largo del proceso productivo y del ciclo de vida de los productos, facilitados fundamentalmente por el internet. ${ }^{15}$

Por lo que al hablar de industria 4.0 conlleva referirnos al uso del internet pues a través de él así como de las diferentes tecnologías de la información y de la comunicación es posible cambiar la forma sustancial de lo que se produce, cómo se trabaja y cómo se vive actualmente borrando las esferas físicas, digitales y biológicas. ${ }^{16}$

De tal suerte que la industria 4.0 es un nuevo modelo de organización hecho posible por la invención de las nuevas tecnologías digitales, el uso de inteligencias artificiales y el internet de las cosas a través de las TIC's permitiendo la creación de productos con mayor eficacia y eficiencia, eliminando las barreras de lo físico y lo digital en los procesos de producción.

En ese contexto la repercusión de la industria 4.0 tanto en el trabajo como en la seguridad social resultan íntimamente ligadas, al menos en México y en

\footnotetext{
tria, España, p. 3, [Consulta: 19 de noviembre, 2019]. Disponible en: http://coddii.org/wp-content/uploads/2016/10/ Informe-CODDII-Industria-4.0.pdf

${ }^{13}$ Comisión Económica para América Latina, Industria 4.0 Oportunidades y desafíos para el desarrollo productivo de la provincia de Santa Fe, CEPAL, Chile, p. 10

${ }^{14} \mathrm{El}$ internet de las cosas es la comunicación entre máquinas por medio de las TIC's capaces de tomar decisiones de manera autónoma sin la intervención de un humano para el desarrollo de determinada actividad.

${ }^{15}$ Basco, Ana Inés, Beliz, Gustavo, et. al., Industria 4.0 fabricando el futuro, UN, Argentina, 2018, p. 24.

${ }^{16} \mathrm{Cfr}$. Ídem.
} 
aquellos países donde la seguridad social se encuentra vinculada al trabajo, por lo que el surgimiento de un nuevo derecho que atienda las nuevas necesidades en el contexto de la industria 4.0 resultan ser necesarias para la protección de las sociedades en estos dos grandes derechos humanos.

\section{La nueva revolución industrial}

La cuarta revolución industrial también conocida como industria 4.0 se encuentra en auge en diversos países del mundo y México no resulta ajeno a este fenómeno global, ciertamente tiene un mayor impacto en naciones con alto desarrollo socioeconómico en comparación a países que se encuentran en vías de desarrollo como es el caso mexicano.

Cabe recordar que la humanidad ha vivido tres grandes revoluciones industriales; la primera, tuvo lugar a finales del siglo XVIII en el Reino Unido misma que se encontró caracterizada por la invención de la máquina de vapor la cual fue la cuna del desarrollo de la época pues tuvo gran auge en los procesos de producción de las industrias así como en los sistemas de comunicación por medio del ferrocarril $;{ }^{17}$ la segunda, aparece a finales del siglo XIX teniendo auge en algunos países en su mayoría europeos. ${ }^{18}$

Esta segunda revolución industrial tuvo un impacto en las industrias ya que se materializó mediante la producción en cadena o masa que permitió a las industrias la elaboración de sus productos con una mayor eficacia y eficiencia reflejándose en el costo y tiempo para producir sus bienes asimismo la creación de medios de transporte como la fabricación del automóvil, buques marítimos de mayor capacidad y el aeroplano impulsado por motores a gasolina. ${ }^{19}$

La tercera revolución industrial tiene inicios en la década de los años setenta teniendo como característica fundamental la invención de las computadoras y el uso del internet así como otros elementos tecnológicos que rompieron paradigmas importantes en materia de comunicación como: los sistemas de navegación a través de satélites y uso de la telefonía remota con la creación de los celulares.

Hoy en día nos encontramos ante la cuarta revolución industrial que trastoca todos los aspectos cotidianos de las personas de una manera totalmente diferente, entre los cuales encontramos: el ámbito laboral y la seguridad social.

\footnotetext{
${ }_{17}$ Mendizábal Bermúdez, Gabriela, Sánchez-Castañeda, Alfredo, et. al, Industria 4.0 Trabajo y seguridad social, IIJUNAM, México, 2019, p. XV.

${ }^{18}$ Paises como Francia, Alemania, Bélgica, Japón y Estados Unidos de América.

${ }^{19}$ Silva Arriaga, Luis, La segunda revolución industrial y el nacimiento de la gran empresa, España, [Consulta: 12 de enero, 2020]. Disponible en: https://ocw.unican.es/pluginfile.php/1213/course/section/1495/MC-II-3.pdf
} 
Una revolución industrial peculiar y distinta que está rompiendo paradigmas en diversos aspectos de la vida de las personas sobre todo por el tipo de tecnologías aplicadas en diversos ámbitos de sus vidas pues son invenciones que nunca se habían visto en la realidad sino solo en películas de ficción.

Esta $4 R I$ ha planteado diversas incógnitas desde su aplicación e impacto a nivel personal, así como la necesidad de los propios Estados de encontrarse preparados para recibir los efectos tanto positivos como negativos que pudieran traer las nuevas tecnologías en el ámbito laboral, económico, social, político y cultural de una sociedad.

Ciertamente los efectos de la $4 R I$ no se sentirán de una manera directa en un corto plazo en países que no tienen la capacidad de desarrollar tecnologías vanguardistas, sin embargo, los efectos permearán de una manera progresiva en todo el mundo en gran medida por el fenómeno de la globalización y la internacionalización.

Se debe mencionar que las revoluciones industriales antecesoras obligaron a las personas ha readaptarse a los nuevos conocimientos y restos que conllevaba la aplicación de las tecnologías, retos que afectaron en su mayoría a los trabajadores de las industrias pues las máquinas llegaron a desplazar puestos de trabajo ocupados por personas, a tal grado que en la Revolución industrial de fines del siglo XVIII y principios del siglo XIX, los trabajadores textiles en el Reino Unido quemaron las máquinas de tejer para protestar contra los nuevos telares automáticos, que habían ocupado sus puestos de trabajo, ${ }^{20}$ por lo que esta nueva revolución industrial no resultaría ser la excepción.

De tal suerte que son hechos que el derecho debe salvaguardar, procurando la protección de dos grandes derechos humanos que han acompañado al hombre desde su existencia: el trabajo y la seguridad social.

\section{La industria 4.0 y el trabajo}

Nuevos trabajos, nuevas profesiones, una reinvención de los trabajadores son algunas de las cosas que depara la industria 4.0 para las personas en materia laboral. La implementación de las tecnologías en el mundo del trabajo ha abierto la posibilidad de la existencia de trabajos que en años anteriores no se creerían, plasmadas en una utopía que hoy se puede hacer realidad.

La incertidumbre se agudiza cuando surgen diversas interrogantes respecto al tema ¿Los robots terminarán realizando los trabajos de las personas? ¿Qué

\footnotetext{
${ }^{20}$ Oppenhaimer, Andrés, Sálvese quien pueda, El debate, México, 2018, p. 44
} 
empleos serán los más afectados por las nuevas tecnologías? ¿Qué se necesita para estar preparados y competir por los empleos del nuevo mercado laboral? ¿Cuál es rol del Estado para la protección de sus ciudadanos en materia de trabajo y seguridad social?

Evidentemente son cuestionamientos sin una respuesta concreta, aunque la realidad es que las tecnologías que conlleva la cuarta revolución industrial impactarán de una manera jamás antes vista en el ámbito laboral, por tanto la reinvención de cada uno de los trabajadores es algo inaplazable.

Existen diversos ejemplos de estos cambios, conscientes de que el impacto y el desarrollo del mismo dependerán del país y las posibilidades tanto económicas como tecnológicas que se disponga, sin embargo, en un mundo donde la globalización ha hecho que todo el planeta se encuentre interconectado, tarde o temprano las tecnologías llegarán a cada uno de los puestos de trabajo incluso a los menos esperados como el caso mexicano.

Cabe destacar que existen centros de distribución de productos que son operados completamente por robots, tiendas completas sin cajeros (Amazon), y restaurantes totalmente automatizados. ${ }^{21}$

La implementación de tecnologías en puestos de trabajo resulta conveniente para los empleadores, ponderando el ahorro en costos laborales que se generarían así como una mayor eficacia y eficiencia en sus productos o servicios, por lo que es muy probable que tiendas, restaurantes, fábricas, centros de distribución, entre otros estén encaminados a la robotización de sus procesos de producción.

De lo anterior se plantea si estas tecnologías pondrán fin al trabajo de las personas. Para lograr responder este cuestionamiento se debe tomar en cuenta lo siguiente:

Se preguntan si la tecnología acabará con el trabajo y con todos los beneficios comúnmente asociados a tener un empleo (por ejemplo, tener acceso a una pensión o seguro médico). Argumentando que estamos viviendo una revolución tecnológica sin precedentes, donde el cambio se está acelerando a un ritmo creciente. Si las máquinas son capaces de ejecutar mejor y de forma más económica lo que hoy hacen los humanos. ¿Qué le espera a la humanidad? ¿Nos condenará la tecnología al desempleo masivo y a la desigualdad? Es obvio que la tecnología tiene

\footnotetext{
${ }^{21}$ Cfr. Pages Serra, Carmen, Ripani, Laura, "El empleo en la Cuarta Revolución Industrial", Revista integración \& comercio, Número 42, agosto, 2017, p. 266.
} 
un enorme potencial de destrucción de empleo, pero ¿cuál será su efecto global? Para anticipar cómo impactará la tecnología al nivel y a la composición del trabajo es preciso tener en cuenta varias cuestiones. ${ }^{22}$

Estas cuestiones se reflejan en lo siguiente: primero, se debe establecer que estas tecnologías podrán remplazar tareas rutinarias es decir, que son fácilmente automatizables, por lo que no todo el trabajo que realizan las personas se verá afectado; segundo, al ser sustituidas las tareas más rutinarias se abre la posibilidad para que profesiones con un grado de preparación mayor se dejen fuera de las máquinas para ser ocupadas por las personas, por lo que una especialización en determinada profesión será requerida para desempeñar esos puestos de trabajo, y; tercero, la creación de nuevos empleos que actualmente no existen, si bien es cierto que algunos desaparecerán o serán ocupados por robots, también resulta verdad que se crearán nuevos trabajos para ser desarrollados por las personas e incluso la implementación de las tecnologías en determinado sector crearan trabajos para poder desempeñar o dar servicio a esas tecnologías, tal y como ha sido documentado por James Bessen, la incorporación masiva de cajeros automáticos no ha acabado con los empleados del sector bancario, sino que, por el contrario, estos han crecido un $2 \%$ por año. ${ }^{23}$

Por lo tanto se desprende que en efecto, las tecnologías de la $4 R I$ impactarán en el mundo del trabajo pero esto no implica la destrucción del mismo sino una trasformación totalmente diferente a la que hoy conocemos, por lo que la acción para contrastar esos efectos se basará en la reinvención de los trabajadores (una nueva reeducación para el trabajo que servirá en el mercado laboral digital) en esta nueva era así como una transformación de lo que conocemos como un trabajo tradicional y las nuevas formas de trabajar.

A pesar de ello se debe abrir el debate respecto del tipo de trabajo que se estará creando sus características y la posible vulneración de los derechos laborales existentes. Al respecto se pude comenzar analizando los siguientes apartados.

\section{a. Las nuevas formas de trabajo en $4 R I$}

Es un hecho que debido a la cuarta revolución industrial las personas deberán estar preparadas para avanzar y realizar cambios en diferentes ámbitos tanto personales como en sociedad. El avance tecnológico no solo trae modificaciones

\footnotetext{
${ }^{22}$ Ibidem, pp. 270-271.

${ }^{23}$ Ibidem, p. 271.
} 
en todo lo que conocemos sino además hace replantearnos si la forma en que estamos haciendo las cosas es la mejor.

El mundo laboral de hace diez años no es el mismo de ahora, ni en México ni en el mundo, la evolución ha sido tan grande que incluso algunos trabajos se han vuelto obsoletos, por lo que el reto es la actualización o reinvención de los trabajadores perfeccionando sus habilidades para estar a la altura de estos cambios y ser sujetos idóneos en el nuevo mercado laboral.

Todos los cambios que han surgido debido al avance tecnológico han traído como resultado nuevas formas de trabajar por ejemplo, en algunos trabajos ya no es necesario estar en un lugar físico para desempeñar la jornada laboral pues gracias a la tecnología podemos estar enviando y recibiendo información desde cualquier lugar.

Estos avances tecnológicos nos dan la oportunidad de realizar el trabajo en cualquier lugar y en el momento que se deseé surgiendo nuevos modelos de trabajo como el Smart Working donde el trabajador podrá decidir en dónde quiere trabajar, teniendo libertad y flexibilidad de horario, asimismo el auge de los FreeLancer, personas que trabajan de manera independiente. Estos dos conceptos nacen y seguirán creciendo gracias a la $4 R I$.

Por consiguiente las nuevas formas de trabajo se pueden ver desde dos perspectivas, por un lado, las grandes empresas buscan personas mejor capacitadas para realizar el trabajo, personas que dominen el tema de las nuevas tecnologías y conozcan cómo utilizarlas con facilidad. Por otro, los empleadores pueden ofertar trabajos fáciles y de tareas sencillas donde el trabajador no necesariamente esté especializado en el área a través de las plataformas digitales, como Uber, Rappi, Didi, entre otros. Por supuesto estos últimos son trabajos más palpables en México.

Por desgracia la creación de estos nuevos empleos conlleva a trabajos precarios ya que en su mayoría son empleos realizados bajo proyecto teniendo como consecuencia que no cuenten con derechos laborales reconocidos en la legislación del trabajo mexicana y por ende, la negación al acceso de seguri-

168 dad social pues no existe una relación laboral sino una prestación de servicios, siendo acreedores únicamente a la percepción económica.

\section{b. Precarización del trabajo}

La llegada de la $4 R I$ ha venido a modificar todo cuanto conocemos; la digitalización, la inteligencia artificial, el desarrollo de las TIC's y las nuevas 
tecnologías no solo han impactado de forma considerable la manera en que vivimos, sino también nos exige adaptarnos y avanzar a la par.

Las sociedades se ha beneficiado con estos procesos tecnológicos en diferentes áreas como la medicina, vivienda, en la creación de productos que nos hacen más fácil la vida, entre otras sin embargo, también trastoca aspectos no muy favorables para la propia sociedad como las modificaciones de los empleos tradicionales, ${ }^{24}$ una precarización de los trabajos existentes que impactan de manera directa a las personas pues estas innovaciones tecnológicas están logrando que los trabajos fácilmente automatizables sean destruidos o remplazables por inteligencias artificiales teniendo como consecuencia que tanto las empresas como las personas que no logren adatarse a este cambio fracasen en un corto plazo.

Todo este cambio tecnológico no es algo nuevo para las personas pues las sociedades siempre se encuentran evolucionando, ellas son capaces de adaptarse y trabajar a la par con los cambios que le marca el propio contexto socio-histórico. Así, los cambios que implica la actual revolución industrial son totalmente nuevos ya que las tecnologías se encuentran en un proceso de independencia, es decir, cada día requieren menos la interacción del ser humano para la toma de decisiones lo cual impacta en las cadenas de producción de las industrias y por ende, los puestos de trabajo serán ocupados en un gran porcentaje por las inteligencias artificiales desplazando a los trabajadores que no sean compatibles con las propias tecnologías que él mismo crea.

Cabe precisar que el miedo al desempleo tecnológico es un fenómeno que ha existido desde hace varios siglos y se ha documentado que se agudiza en épocas de cambios tecnológicos radicales ${ }^{25}$ como ya lo estamos viviendo.

Además, la cuarta revolución industrial va más allá de inteligencia artificial o del uso de robots, este avance tecnológico está trayendo nuevas formas de trabajo, desempleo, transformación, entre otros puntos repercutiendo directamente en las personas, su economía y en su aseguramiento social.

Sin duda alguna replantearse el desempleo como una posibilidad de este hito tecnológico resulta ser una constante ya que por un lado, las grandes empresas buscarán la forma de producir más gastando menos, lo cual implica invertir en máquinas que puedan realizar lo mismo que diez trabajadores, las

\footnotetext{
${ }^{24}$ El periodista Andrés Oppenheimer en su obra ¡Sálvese quien pueda! El futuro del trabajo en la era de la automatización hace una descripción de cómo los trabajos tradicionales como los banqueros, mensajeros, operadores de cabina entre otros desaparecerán producto de las máquinas y las tecnologías de esta era.

${ }^{25}$ Minian, Isaac y Martínez Monroy, Ángel, "El impacto de las nuevas tecnologias en el empleo en México", Revista Problemas del Desarrollo, octubre-diciembre, 2018, p. 28.
} 
cuales no requieren aportaciones a la seguridad social, no se enferman, no existe una relación laboral, ni mucho menos prestaciones laborales.

De tal suerte que las personas podrán ser remplazadas por máquinas en sus puestos de trabajo. ${ }^{26}$

El mundo laboral está cambiando, hoy en día el aumento de los empleos a través de plataformas digitales y trabajos bajo la modalidad de proyectos en México ${ }^{27}$ son ocupados por los jóvenes que se encuentran interesados en desempeñar estos servicios ya que por un lado pueden desarrollar la actividad laboral en el lugar que ellos decidan si tener que acudir a un espacio físico para realizarlas y por supuesto no estar ligados a un horario laboral.

Pese a ello se debe mencionar que carecen de alguna certeza o permanencia en el empleo debido a que estas formas de trabajar en México son desarrolladas como prestación de servicios regidas por legislación de corte civil, lo que implica que solo tendrán derecho a los honorarios recibidos por la contraprestación excluyendo las prestaciones laborales como: vacaciones, aguinaldos, aseguramiento social, entre otros.

Estas formas precarizadas de trabajar son el auge hoy en día, trabajos que se encuentran formando a los nuevos jornaleros digitales de la nueva era. ${ }^{28}$

El trabajo tradicional podrá encontrarse en vías de extinción dando paso a estas formas de trabajar que desgraciadamente vulnera los derechos laborales que a lo largo de los años han sido objeto de lucha de las y los trabajadores y han permitido un equilibro social.

Asimismo el derecho a la seguridad social deberá adaptarse a las nuevas condiciones y situaciones que le exige el contexto social con la finalidad de continuar brindando esa protección que requieren las personas de una sociedad.

\section{El derecho a la seguridad social frente a la industria 4.0}

El mundo del trabajo se encuentra rompiendo paradigmas y transformándose a formas de trabajar totalmente diferentes a las tradicionales por lo tanto, el

\footnotetext{
${ }^{26}$ Economía Basada en Recursos, El Desempleo Tecnológico: Conceptos EBR, Colombia, 2015, [Consulta: 14 de septiembre, 2019]. Disponible en: http://economiabasadaenrecursos.co/index.php/economia-basada-en-recursos/conceptos-ebr/desempleo-tecnol\%C3\%B3gico.html

${ }^{27} \mathrm{Cfr}$. Garcia, Ana Karen, Precariedad laboral en plataformas está creando jornaleros digitales: OIT, El economista, 2019, [Consulta: 14 de septiembre, 2019]. Disponible en: https://factorcapitalhumano.com/innovacion-y-tecnologia/precariedad-laboral-en-plataformas-esta-creando-jornaleros-digitales-oit/2019/02/

${ }^{28}$ Cfr. Millán, Mauricio, Empleo calificado ante la Cuarta Revolución Industrial, Vanguardia, México, 2018, [Consulta: 12 de noviembre, 20109]. Disponible en: https://vanguardia.com.mx/articulo/empleo-calificado-ante-la-cuarta-revolucion-industrial
} 
sistema jurídico mexicano debe adaptarse a las nuevas realidades sociales para regular y otorgar una seguridad jurídica a las personas en todos los ámbitos donde el derecho se vea involucrado.

Si el trabajo está cambiando también la forma de brindar seguridad social debe cambiar y adaptarse a los nuevos contextos y necesidades que marcan las situaciones de la vida cotidiana de una sociedad, tomando como premisa que la seguridad social es un instrumento de politica social que se diseña para responder a las necesidades de una colectividad en un tiempo determinado. ${ }^{29}$

Cabe mencionar que la materialización de la seguridad social resulta un tema sumamente complejo pues otorgar este derecho humano ${ }^{30}$ en países en vías de desarrollo es complicado, en primer lugar por la falta de reconocimiento de la seguridad social como un derecho humano y; segundo, al no establecer los medios idóneos para poder hacer efectivo este derecho a toda la población, limitándose a solo brindar una protección social a través del asistencialismo.

Actualmente (al menos en lo que respecta a México) el requisito indispensable de contar con un trabajo formal para acceder a la seguridad social resulta una limitante máxime por el gran número de personas que se encuentran en el sector informal superando a aquellas que se encuentran en la formalidad.

Por lo que la barrera de la formalidad laboral tendrá que modificarse para abrir otros mecanismos de aseguramiento social que no solo se encuentren ligados a la formalización de un empleo.

Con ello se abre la interrogante respecto de ¿por qué la industria $4.0 \mathrm{im}-$ pacta en la seguridad social? Pues bien, como se ha mencionado con anterioridad todas las innovaciones tecnológicas traen consigo ventajas y desventajas y esta $4 R I$ no es la excepción.

Por lo que respecta a las ventajas, podemos establecer que existe una mayor producción con mayor eficacia y eficiencia, reducción de costos de elaboración, flexibilización en las jornadas laborales permitiendo una conciliación de la vida laboral-familiar así como nuevas invenciones que permiten realizar la vida cotidiana más fácil de una persona.

Sin embargo, las desventajas también se encuentran presentes en esta industria 4.0 siendo más notorias y perjudiciales para la clase trabajadora e incluso, para cualquier persona que tenga una relación laboral ya que se encuentra en juego su aseguramiento social en razón a que las inteligencias artificiales son capaces de ocupar puestos de trabajo que son fácilmente automatizables,

\footnotetext{
${ }^{29}$ Narro Robles, José, Moctezuma Navarro, David, et al., "Hacia un nuevo modelo de seguridad social", Economía UNAM, vol. 7, núm. 20 mayo-agosto 2010.

${ }^{30}$ En el caso mexicano desconoce dentro de su carta magna el Derecho a la Seguridad Social como derecho humano.
} 
lo que traería como consecuencia una precarización en el aseguramiento social de las personas así como el aumento en la tasa de desempleo y una alza en los trabajos de la economía informal. ${ }^{31}$

Si bien es cierto, no todos los trabajos serán ocupados por las máquinas, pero podrá existir una precarización en las jornadas laborales debido a la posibilidad de una reducción de las mismas, pues las máquinas podrán absorber tiempos de trabajo que realizaban las personas cuestión más redituable para el patrón.

Tal precarización de las jornadas de trabajo tendrá como efecto una precarización en el aseguramiento social de cada una de las y los trabajadores.

En ese contexto estos cambios tecnológicos en el mundo del trabajo tienen un impacto en los sistemas de seguridad social ya que el aumento de desempleo trae consigo la pérdida de acceso a ésta y por desgracia, la laboralización de la seguridad social en México resulta ser una problemática constante.

De tal suerte que las politicas de protección social deben adaptarse a los cambios en el mundo laboral. ${ }^{32}$

También algunos trabajadores y los propios empleadores se verán beneficiados con los avances tecnológicos de esta revolución industrial aunque, existirá una mayor afectación a las y los trabajadores derivado del peligro de ser sustituidos de los puestos de trabajo por las máquinas.

De este modo es necesaria una transformación de la seguridad social que responda a los requerimientos y necesidades que demanda una sociedad 4.0 carente de mecanismos de protección social frente a las nuevas invenciones tecnológicas en el nuevo mercado laboral.

\section{Hacia una seguridad social 4.0}

La premisa de lo anteriormente analizado es que el trabajo tradicional que hoy conocemos sufrirá grandes cambios a consecuencia de la industria 4.0, por lo que es congruente mencionar que el sistema de seguridad social de México debe de cambiar máxime aquellos que establecen que para generar el derecho a la seguridad social es indispensable la existencia de un trabajo formal.

\footnotetext{
${ }^{31} \mathrm{Cfr}$. Mendizábal Bermúdez, Gabriela, López Pérez, Emmanuel, "¿Un nuevo modelo de seguridad social?", Revista Internacional y Comparada de Relaciones laborales y Derecho del Empleo, Volumen 6, núm. 1, enero -marzo, 2018, pp. 298-327.

${ }^{32}$ Banco Mundial, Las políticas de protección social deben adaptarse a los cambios en el mundo laboral, dice el Banco Mundial, Banco mundial, Estados Unidos de América, 2019, [Consulta: 23 de noviembre, 2019]. Disponible en: https://www.bancomundial.org/es/news/press-release/2019/09/17/social-protection-must-adapt-to-changingnature-of-work-world-bank-says
} 
Continuar con los sistemas tradicionales de aseguramiento social traerían una posible precarización en materia de protección social de las personas que hoy en día se encuentran vinculadas a la seguridad social sin embargo, surge la incertidumbre de que los trabajos que actualmente se encuentran activos, en unos años puedan dejar de existir o en el mejor de los casos se trasformen y que cambien las condiciones de contratación del empleado (a), pasando de una relación de trabajo personal y subordinada para actualizarse a una simple prestación de servicios profesionales, originando que el trabajador (a) (futuro profesionista) así como sus beneficiarios pierdan el derecho a acceder a la seguridad social bajo un régimen obligatorio dejándolos en un estado de vulnerabilidad.

De lo anterior se desprende la necesidad de plantearse una transformación de los sistemas de protección social para formar una seguridad social 4.0.

En ese contexto, cabe destacar lo siguiente:

Seguridad social 4.0 es el conjunto de programas sociales (PPS) seguros sociales (prestaciones de derecho laboral) y servicios obligatorios de carácter público y privado que, integrados en una red y con diversos mecanismos de exigibilidad jurídica, positivicen y materialicen el derecho humano a la seguridad social en una sociedad regida por la industria $4.0 .^{33}$

La seguridad social 4.0 es la integración de las propias herramientas de la seguridad social pasando por aquellas que deriven de la asistencia social, los seguros sociales y los seguros voluntarios.

Esta integración debe estar debidamente relacionada entre sí y no ser manejada como instrumentos totalmente diferentes, debe existir una articulación entre ellas para poder perseguir un objetivo en común, el máximo otorgamiento de protección social a las personas.

En ese sentido el Banco Mundial en su informe sobre el desarrollo Mundial 2019 "La naturaleza cambiante del trabajo" establece que:

Los tres principales componentes de los sistemas de protección social (un mínimo social garantizado -con la asistencia social como eje principal, la seguridad social y la regulación del mercado de trabajo) pueden servir para abordar los desafíos que presenta el mercado laboral. ${ }^{34}$

\footnotetext{
${ }^{33}$ Mendizábal Bermúdez, Gabriela, Sánchez-Castañeda, Alfredo, et al, (coord.), op. cit., p. 11.

${ }^{34}$ Traducción personal a partir de: three main components of social protection systems-a guaranteed social minimum (with social assistance at its core), social insurance, and labor market regulation-can manage labor market
} 
De esta aseveración, se puede concluir que la tendencia en materia de protección social es el establecimiento de pisos de protección social en cada uno de los Estados, una iniciativa liderada por la Organización de las Naciones Unidas y la Organización Internacional del Trabajo que considera la provisión de servicios esenciales y transferencias a todas aquellas personas en necesidad de protección para prevenir que caigan en estado de pobreza extrema o facilitar su salida de la misma..$^{35}$ Aunque se debe poner peculiar atención en el tema ya que para el establecimiento de PPS ${ }^{36}$ deben atenderse las propias recomendaciones de la OIT para una correcta aplicación del mismo en la sociedad.

A través del PSS se pretende establecer un mínimo de protección social, la cual:

Incluye un conjunto de programas de asistencia social que brindan apoyo financiero a una gran parte de la población, o incluso a su totalidad. Impulsada por la preocupación por la equidad, la expansión de la asistencia social cobra relevancia en vista de los crecientes riesgos que surgen en los mercados laborales y dada la importancia de brindar un apoyo adecuado, independientemente del modo en que la persona participa en esos mercados.

\section{$(\ldots)$}

El objetivo de este enfoque es ampliar la cobertura dando a la vez prioridad a los más pobres. ${ }^{37}$

Sin embargo, se debe estar conscientes que la asistencia social debe complementarse con un seguro que no dependa exclusivamente del empleo asalariado formal. Un sistema de este tipo permitiría brindar cobertura básica universal, subsidiando las primas para los pobres y extendiendo la asistencia social, ${ }^{38}$ pero al mismo tiempo debe contemplarse el establecimiento de contribuciones obligatorias con base a los ingresos de las personas.

challenges, en: Worl Bank, The changing nature of work, World bank Group, 2019, Estados Unidos de América, p. 106.

${ }^{35}$ Bertranou, Fabio, Iniciativa del Piso de Protección Social, Ed. OIT, 2010, p. 1, [Consulta: 20 de enero, 2020]. Disponible en: http://www.ilo.org/wcmsp5/groups/public/---ed_protect/---soc_sec/documents/publication/wcms_secSoc_17458.pdf

${ }^{36}$ Piso de Protección Social

${ }^{37}$ World Bank, op. cit., p. 106.

${ }^{38}$ ídem. 
Una seguridad social 4.0 requiere grandes cambios en los sistemas de seguridad social de los Estados así como una concientización de las personas en relación a la importancia de afiliarse a dichos sistemas de seguridad social. Una estrategia integral de promoción de la cultura entre los actores que intervengan en ella impulsando el aseguramiento social a través de seguros sociales en colaboración con los demás instrumentos, sino se tiene claro los beneficios que acarrea contar con seguridad social no se va a avanzar mucho; si sabemos cuáles son las prestaciones económicas que se derivan de ella, la vamos a valorar, ${ }^{39}$ máxime en esta era tan cambiante que repercute en el día a día de las personas y su entorno familiar.

\section{Reflexiones finales}

Estas reflexiones finales nos lleva a desarrollarlas en dos puntos clave:

1. El ámbito laboral. Es un hecho que el mundo del trabajo está cambiando exponencialmente y nuestra profesión también por lo tanto, una reinvención del trabajador resulta ser una necesidad que se debe atender en México y en el resto del mundo.

La reeducación de las personas tiene que ser base elemental para no quedar desfasados ante esta oleada tecnológica que acarrea la $4 R I$ para su incorporación al nuevo mercado laboral a través del desarrollo de competencias habilitantes para la industria 4.0.

2. Seguridad social. Es claro que este derecho debe de ajustarse a una nueva realidad que permita el acceso a sus prestaciones a todas las personas, para ello, es indispensable hablar de una deslaboralización de la seguridad social es decir, establecer otros mecanismos de acceso diferentes al trabajo formal.

Por supuesto una construcción de un modelo de seguridad social donde intervengan de manera articulada sus propios instrumentos: asistencia social, seguros sociales y seguros privados.

\footnotetext{
${ }^{39}$ Secretaria del Trabajo y Previsión Social, Fomenta STPS cultura de la Seguridad Social, Gobierno de México, México, 2017, [Consulta: 19 de enero, 2020]. Disponible en: https://www.gob.mx/stps/prensa/fomenta-stps-cultura-de-la-seguridad-social
} 
Por último, atendiendo que hoy en día los mecanismos de protección social fungirán como medios de contención para la protección de contingencias en esta era tan cambiante, resulta necesario establecer una concientización de la importancia de este derecho en la vida de las personas por lo que una culturalización de la seguridad social como formación de vida debe ser implementada en cada una de las sociedades.

\section{Fuentes de investigación}

Banco Mundial, Las políticas de protección social deben adaptarse a los cambios en el mundo laboral, dice el Banco Mundial, Banco mundial, Estados Unidos de América, 2019, [Consulta: 23 de noviembre, 2019]. Disponible en: https://www.bancomundial.org/es/news/press-release/2019/09/17/ social-protection-must-adapt-to-changing-nature-of-work-world-bank-says

Basco, Ana Inés, Beliz, Gustavo, et al., Industria 4.0 fabricando el futuro, UN, Argentina, 2018, p. 24.

Bertranou, Fabio, Iniciativa del Piso de Protección Social, Ed. OIT, 2010, p. 1, [Consulta: 20 de enero, 2020]. Disponible en: http://www.ilo.org/wcmsp5/groups/public/--ed_protect/---soc_sec/documents/publication/wcms_secsoc_17458.pdf

Briceño Ruiz, Alberto, Derecho de la seguridad social, Ed. Oxford, México, 2011, p. 14. --_--, Derecho Mexicano de los Seguros Sociales, Ed. Harla, México, 2017, p. 13.

Castresna Sáenz, Carolina, et al., Industria 4.0, España, Universidad de la Rioja, 2016, p. 12, [Consulta: 22 de diciembre, 2019]. Disponible en: https://biblioteca.unirioja. es/tfe_e/TFE002004.pdf

Comisión Económica para América Latina, Industria 4.0 Oportunidades y desafíos para el desarrollo productivo de la provincia de Santa Fe, CEPAL, Chile, p. 10

Conferencia de Directores y Decanos de Ingeniería Informática, Industria 4.0: la transformación digital de la industria, España, p. 3, [Consulta: 19 de noviembre, 2019]. Disponible en: http://coddii.org/wp-content/uploads/2016/10/ Informe-CODDII-Industria-4.0.pdf

De Buen, Néstor, Derecho del Trabajo, Editorial Porrúa, México, 2015, p. 19.

Díaz, Verónica, Revolución Industrial 4.0, inflexión para la fuerza laboral, Prensario TI Latin América, Argentina, 2017, en: https://www.prensariotila.com/19524-Revolucion-Industrial-40-inflexion-para-la\%20fuerzalaboral.note.aspx

Economía Basada en Recursos, El Desempleo Tecnológico: Conceptos EBR, Colombia, 2015, [Consulta: 14 de septiembre, 2019]. Disponible en: http://economiabasadaenrecursos.co/index.php/economia-basada-en-recursos/conceptos-ebr/ desempleo-tecnol\%C3\%B3gico.html

Forbes, Globalización 4.0: una transformación constante, México, 2019, [Consulta: 23 de diciembre, 2019]. Disponible en: https://www.forbes.com.mx/ globalizacion-4-0-una-transformacion-constante/ 
García, Ana Karen, Precariedad laboral en plataformas está creando jornaleros digitales: OIT, El economista, 2019, [Consulta: 14 de septiembre, 2019]. Disponible en: https://factorcapitalhumano.com/innovacion-y-tecnologia/precariedad-laboral-en-plataformas-esta-creando-jornaleros-digitales-oit/2019/02/

Ley del Seguro Social, Diario Oficial de la Federación, 21 de diciembre de 1992.

Ley Federal del Trabajo, Diario Oficial de la Federación, 1 de abril de 1970.

Mendizábal Bermúdez, Gabriela, López Pérez, Emmanuel, “¿Un nuevo modelo de seguridad social?”, Revista Internacional y Comparada de Relaciones laborales y Derecho del Empleo, Volumen 6, núm. 1, enero -marzo, 2018, pp. 298-327.

Mendizábal Bermúdez, Gabriela, Sánchez-Castañeda, Alfredo, et al, Industria 4.0 Trabajo y seguridad social, IIJ-UNAM, México, 2019, p. XV.

Millán, Mauricio, Empleo calificado ante la Cuarta Revolución Industrial, Vanguardia, México, 2018, [Consulta: 12 denoviembre, 20109].Disponibleen:https://vanguardia.com.mx/articulo/empleo-calificado-ante-la-cuarta-revolucion-industrial

Minian, Isaac, Martínez Monroy, Ángel, “El impacto de las nuevas tecnologías en el empleo en México”, Revista Problemas del Desarrollo, octubre-diciembre, 2018, p. 28.

Naciones Unidas, Declaración Universal de los Derechos Humanos, 1948, en: https:// www.un.org/es/universal-declaration-human-rights/

Narro Robles, José, Moctezuma Navarro, David, et al., "Hacia un nuevo modelo de seguridad social”, Economía UNAM, vol. 7, núm. 20 mayo-agosto 2010.

OBS Business School, Industria 4.0, entonces Formación 4.0, Universitat Barcelona, España, [Consulta: 30 de septiembre, 2019]. Disponible en: https://www.obs-edu. com/int/blog-investigacion/logistica/industria-40-entonces-formacion-40

Oppenhaimer, Andrés, Sálvese quien pueda, El debate, México, 2018, p. 44

Organización Internacional del Trabajo, ¿Qué es el trabajo decente?, OIT, Suiza, 2004, [Consulta: 4 de enero, 2020]. Disponible en: https://www.ilo.org/americas/sala-de-prensa/WCMS_LIM_653_SP/lang--es/index.htm

Organización Internacional del Trabajo, Indicadores del sistema seguridad social glosario de seguridad social y términos relacionados, Ed. OIT, Perú, 2012, [Consulta: 13 de diciembre, 2019] Disponible en: http://white.lim.ilo.org/spanish/260ameri/oitreg/activid/proyectos/actrav/segsoc/estadisticas/indicadores/glosario. html

Organización para la Cooperación y el Desarrollo Económicos, El cambiante mundo laboral requiere una nueva estrategia de empleo, dice la OCDE, OCDE, 2018, [Consulta: 19 de octubre, 2019]. Disponible en: https://www.oecd.org/centrodemexico/medios/elcambiantemundolaboralrequiereunanuevaestrategiadeempleodicelaocde.htm

Pages Serra, Carmen, Ripani, Laura, "El empleo en la Cuarta Revolución Industrial”, Revista integración \&t comercio, Número 42, agosto, 2017, p. 266.

Paramo López, Paola y Díaz Lozano, M. José, Economía: Ventajas y desventajas de la globalización, La verdad, España, 2019, [Consulta: 1 de noviembre, 2019]. 
Disponible en: http://www.miperiodicodigital.com/2019/grupos/pamfas-77/ economia-ventajas-desventajas-globalizacion-2107.html

Secretaría del Trabajo y Previsión Social, Fomenta STPS cultura de la Seguridad Social, Gobierno de México, México, 2017, [Consulta: 19 de enero, 2020]. Disponible en: https://www.gob.mx/stps/prensa/fomenta-stps-cultura-de-la-seguridad-social

Silva Arriaga, Luis, La segunda revolución industrial y el nacimiento de la gran empresa, España, [Consulta: 12 de enero, 2020]. Disponible en: https://ocw.unican.es/ pluginfile.php/1213/course/section/1495/MC-II-3.pdf

World Bank, The changing nature of work, World Bank Group, 2019, Estados Unidos de América, p. 106. 